\title{
Características clínico-epidemiológicas de pacientes con cólera en la ciudad de México
}

\author{
A gustina Elena Vilchis-Guizar, M.C., (1) Samuel U ribe-Márquez, M.C., (2)
}

Patricia Leonor Pérez-Sánchez, M.C., M. en C.(3)

\begin{abstract}
Vilchis-Guizar AE, Uribe-Márquez S, Pérez-Sánchez PL.

Características clínico-epidemiológicas de pacientes con cólera en la ciudad de México. Salud Publica Mex 1999;41:487-491.
\end{abstract}

\begin{abstract}
Resumen
Objetivo. Comparar las características clínico-epidemio lógicas de enfermos de cólera (casos) con las de pacientes con diar rea por otra causa (no casos), atendidos en las unidades médicas de la Delegación $2 \mathrm{~N}$ oreste del Distrito Federal, del Instituto Mexicano del Seguro Social (IMSS). Material y métodos Se realizó un estudio transversal analítico y se utilizaron datos de 4952 notificaciones reunidas entre 1995 y 1998 a través del formato oficial "Hoja de notificación inmediata de casos de cólera", que cada unidad médica envía a la C oordinación de Salud Comunitaria del IMSS. Mediante estadística analítica se compararon medias y proporciones para ambos grupos. Resultados Se identificaron 588 pacientes con cólera y 4364 con diarrea por otra causa. En el primer grupo, la edad promedio varió entre los 39 y los 51 años, y predominó el sexo femenino (54\%). Durante 1995 y 1997 (años en los que se presentó un número más elevado de casos), los pacientes de cólera, en relación con el grupo de comparación, presentaron una mayor frecuencia de evacuaciones líquidas (97 vs. 73\%), con aspecto de "aqua de arroz" (31 vs. $13 \%$ ), vó mitos (72 vs. $63 \%$ ), espasmos musculares (49 vs. $26 \%$ ), deshidratación (83 vs. $71 \%$ ), choque hipo volémico $(10$ vs. $1 \%)$ y defunciones $(0.85 \%$ vs. $0.25 \%)$, con diferencias estadísticamente significativas. Conclusiones El cólera muestra un comportamiento cíclico bianual; a mayor frecuencia mayor gravedad de la enfermedad. Se requiere incrementar la vigilancia epidemioló gica y el esfuerzo médico para un diagnóstico y tratamiento oportuno.
\end{abstract}

Palabras clave: cólera; diarrea; México
Vilchis-Guizar AE, Uribe-Márquez S, Pérez-Sánchez PL.

Clinical and epidemiological characteristics

of cholera patients in Mexico City. Salud Publica Mex 1999;41:487-491.

\begin{abstract}
A bstract
Objective. To compare the clinical and epidemiological characteristics of cholera patients and patients with diarrhea of different etiology (controls), treated at Mexican Institute of So cial Security medical units in N ortheast Mexico City, from 1995 to 1998. Material and methods Crosssectional analytical study design. Data were collected using the official form "Immediate N otification of Cholera Cases" that each medical unit reports to the Coordination of Co mmunity H ealth. Statistical analysis consisted of comparisons of means and proportions between both groups. $\mathbf{R e}$ sults In 4952 notifications, there were 588 cholera cases and 4364 controls. The age range of cases was 39 to 51 years and 54\% were females. During 1995 and 1997 (years with higher proportion of cases) patients with cholera had a greater frequency of watery evacuations than controls $(97 \%$ vs. $73 \%)$, "rice water" appearance (31\% vs. $13 \%)$, vomiting ( $72 \%$ vs. $63 \%)$, muscular spasms ( $49 \%$ vs. $26 \%)$, dehydration $(83 \%$ vs. $71 \%)$, hypovolemic shock ( $10 \%$ vs. $1 \%)$ and death $(0.85 \%$ vs. $0.25 \%)$. These differences were statistically significant. Conclusions Cholera presents a biannual cyclic behavior; greater frequencies are associated with greater severity and complications. It is necessary to increase epidemiologic surveillance and medical efforts for opportune diagnosis and treatment.
\end{abstract}

Key words: cholera; diarrhea; Mexico

(1) Profesora del diplomado de Epidemiología Clínica, D elegación 2 N oreste D istrito Federal (D.F.), Instituto Mexicano del Seguro Social (IMSS), México.

(2) Coordinador delegacional de Salud C omunitaria, Delegación 2 N oreste D.F., IMSS, México.

(3) Coordinadora delegacional de Investigación Médica, D elegación 2 N oreste D.F., IMSS, México.

Fecha de recibido: 1 de octubre de 1998 - Fecha de aprobado: 23 de junio de 1999

Solicitud de sobretiros: D ra. A gustina ElenaVilchis Guizar. 0 riente 154, núm. 171, colonia Moctezuma, 15500 México, D.F., México. 
E 1 cólera es una infección intestinal aguda causada por el Vibrio cholerae (V. cholerae), y se caracteriza por la aparición brusca de diarrea abundante, vómitos, deshidratación e, incluso, colapso circulatorio; no recibir atención inmediata, puede llevar a la muerte en pocas horas. ${ }^{1,2} \mathrm{El}$ primer caso mexicano de la actual pandemia se notificó en junio de 1991, en el Estado de México. ${ }^{2-5}$ Desde entonces y hasta el 31 de diciembre de 1998, se confirmaron 45963 casos en todo el país, con 552 defunciones $(1.2 \%) ;{ }^{6}$ del total de casos, el Instituto Mexicano del Seguro Social (IMSS) atendió 22418 (49\%) y notificó 269 defunciones (1.2\%). ${ }^{7} \mathrm{La}$ figura 1 muestra la frecuencia de casos de cólera ocurridos desde junio de 1991 hasta diciembre de 1998.

La enfermedad se presenta por casos importados, en casos aislados, como de lenta evolución o como epidemia explosiva. ${ }^{3,4}$ Aunque se han descrito más de 60 serogrupos de $V$. cholerae, sólo el 01 ocasiona la enfermedad; en el continente americano, en particular, el biotipo Tor es el que predomina. El serotipo Inaba destacó por su frecuencia durante los primeros años, pero fue rápidamente remplazado por el Ogawa tanto en nuestro país como en el resto de América Latina, el cual ha sido aislado en la mayoría de los casos que se ha presentado últimamente. ${ }^{3,8-10}$

El cuadro clínico puede variar, pues bien pueden presentarse casos asintomáticos o con diarrea leve, al igual que pacientes con la forma más grave de la enfermedad, es decir, con falta de apetito, malestar abdominal, abundante diarrea líquida -que se torna blanquecina y semeja agua de arroz-, vómitos frecuentes, fiebre baja o casi inexistente, y espasmos abdominales y musculares. ${ }^{3,4,8}$ La deshidratación es la

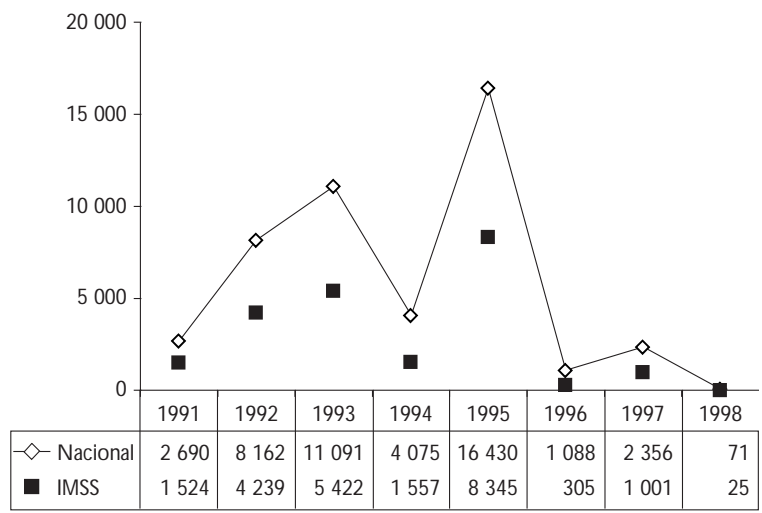

Figura 1. Comportamiento epidemiológico del Cólera en el ámbito nacional y en el Instituto Mexicano del Seguro Social. México, 1991-1998 complicación más frecuente y grave, por lo que el tratamiento debe basarse en la reposición de líquidos, aunada al empleo de doxiciclina para disminuir la duración y el volumen de la diarrea, así como el tiempo de excreción de la bacteria., ${ }^{3,4}$

En México, diversas investigaciones refieren las características y la evolución de los pacientes con cólera, ${ }^{2,11-14}$ sobre todo de los brotes epidémicos de los primeros años; sin embargo, la literatura acerca de las características clínicas y epidemiológicas del padecimiento, a lo largo de un mayor periodo, es escasa. Además, poco se han comparado las características de los pacientes que padecen cólera, con las de aquellos que cursan con enfermedades enterales de otro tipo; por ello, el objetivo del presente trabajo fue identificar las diferencias epidemiológicas y clínicas que había entre enfermos con cólera y pacientes que cursaban con diarrea de otra etiología.

\section{Material y métodos}

El diseño de la investigación fue transversal y analítico. La población de estudio incluyó a pacientes con cuadro diarreico, atendidos en las unidades médicas de la Delegación 2 Noreste del IMSS, en el Distrito Federal (dicha delegación comprende la zona norte y noreste del área metropolitana de la ciudad de México). A cada paciente se le realizó un cultivo de $V$. cholerae en heces o vómito para confirmar o descartar la presencia de cólera.

La fuente de información fue el formato oficial del IMSS, denominado "Hoja de notificación inmediata de casos de cólera", el cual se utilizó para hacer el diagnóstico de los pacientes con cuadro enteral (sugestivo o no de cólera) atendidos en las unidades médicas. Este formato, junto con el resultado del cultivo, se envió a la Coordinación Delegacional de Salud Comunitaria. Cabe aclarar que, si se confirmaba un caso de cólera, se tomaban cultivos en los contactos intradomiciliarios.

Sobre la base de estas notificaciones se formaron dos grupos: el primero estaba integrado por pacientes en los que se confirmó cólera (casos), y el segundo, por sujetos en los que se descartó el padecimiento (no casos). En ambos grupos se identificaron características epidemiológicas, cuadro clínico, presencia de deshidratación y/o choque hipovolémico, así como mortalidad.

El análisis de los datos comprendió estadística descriptiva y analítica, y se comprararon las características de los casos y no casos mediante la prueba $t$ de Student y $\chi^{2}$ (de acuerdo con el nivel de medición de las variables). 
El protocolo fue aprobado por un comité local de investigación del IMSS, en una de las unidades médicas participantes.

\section{Resultados}

Del 1 de enero de 1995 al 31 de diciembre de 1998, se recibieron 4952 notificaciones; se confirmaron 588 casos de infección por cólera (469, 7, 110 y 2, para 1995, 1996, 1997 y 1998, respectivamente) y 4364 pacientes sin el padecimiento $(1910,850,732$ y 872, para los mismos años). En 583 casos de cólera se aisló $V$. cholerae 01 Ogawa (99\%), y en cinco, V. cholerae 01 Inaba $(1 \%)$.

Todos los pacientes eran derechohabientes del IMSS, aunque no siempre adscritos a unidades médicas de la Delegación 2 Noreste. Las delegaciones políticas con mayor número de pacientes con cólera fueron: Gustavo A. Madero (27\%), Cuauhtémoc (21\%), Venustiano Carranza (16\%) y el municipio de Nezahualcóyotl, Estado de México (25\%).

En el grupo de pacientes con cólera, había 316 mujeres ( $54 \%)$, y en el de no casos, 2562 (59\%) ( $p>0.05)$. La edad de los primeros oscilaba entre los cuatro meses y los 95 años, y la de los segundos, entre los seis meses y los 97 años. El promedio de edad fue, durante 1995 y 1997, mayor en los pacientes con cólera (46 años vs. 38 y 51 años vs. 42 , respectivamente, $p<0.001$ ), al igual que el consumo de alimentos en la vía pública ( $51 \%$ vs. $45 \%, p<0.05$ en 1995 y 1996) (cuadro I).

Los pacientes con cólera, respecto a aquellos que no tenían el padecimiento, durante 1995 y 1997 mostraron una mayor frecuencia ( $97 v s .73 \%$ ) y promedio
(15 vs. 12 y 16 vs. 13) de evacuaciones líquidas, mayor presencia de heces con aspecto de "agua de arroz" (31 vs. $13 \%)$, mayor frecuencia (72 vs. 63\%) y promedio (8 vs. 4 y 5 vs. 4) de vómitos, espasmos musculares ( 49 vs. $26 \%)$, deshidratación leve y moderada (83 vs. $71 \%$ ), choque hipovolémico (10 vs. $1 \%$ ) y defunciones $(0.85 \%$ vs. $0.25 \%$ ). Estas situaciones no se observaron durante 1996 y 1998, años en los que hubo un menor número de casos. Excepto para defunción, las diferencias entre ambos grupos tuvieron significancia estadística en 1995 y 1997, no así en 1996 y 1998 (cuadro II).

\section{Discusión}

El cólera es ya una enfermedad endémica en nuestro país. El presente trabajo mostró que, durante 1995 y 1997, su incidencia tuvo un notable incremento respecto a 1994 y 1996, lo que pone de manifiesto su comportamiento cíclico, el cual se ha considerado, en parte, secundario a variaciones en el comportamiento ecológico de la bacteria. ${ }^{10}$

Las características epidemiológicas del cólera muestran que esta enfermedad puede presentarse en cualquier etapa de la vida, pero que afecta sobre todo a la población adulta que se encuentra entre la cuarta y la quinta década de vida; este hallazgo es similar al obtenido por otros investigadores, cuyos pacientes, en su mayoría, se han ubicado entre los 25 y los 45 años de edad. .11, 13

En cuanto al sexo, en forma global se observa un discreto predominio de las mujeres, tanto en los casos de cólera como en los no casos; otro autores como Pastrana Huamnaco ${ }^{11}$ en Lázaro Cárdenas, Michoacán, y

\section{Cuadro I}

Características epidemiológicas en los paCientes Con Y Sin diagnóstico de Cólera. Delegación 2 Noreste del Distrito Federal, Instituto Mexicano del Seguro Social, 1995-1998

\begin{tabular}{|c|c|c|c|c|c|c|c|c|}
\hline \multirow[t]{2}{*}{ Características } & \multicolumn{2}{|c|}{1995} & \multicolumn{2}{|c|}{1996} & \multicolumn{2}{|c|}{1997} & \multicolumn{2}{|c|}{1998} \\
\hline & Con cólera & Sin cólera & Con cólera & Sin cólera & Con cólera & Sin cólera & Con cólera & Sin cólera \\
\hline N úmero & 469 & 1910 & 7 & 850 & 110 & 732 & 2 & 872 \\
\hline Edad* & $46 \pm 21^{\S}$ & $38 \pm 20$ & $39 \pm 27$ & $44 \pm 33$ & $51 \pm 20^{\S}$ & $42 \pm 21$ & $37 \pm 6$ & $40 \pm 18$ \\
\hline \multicolumn{9}{|l|}{ Sexo $0^{\ddagger}$} \\
\hline Masculino & $216(46 \%)$ & $825(43 \%)$ & $5(71 \%)$ & $340(40 \%)$ & $49(45 \%)$ & $291(40 \%)$ & $2(100 \%)$ & $334(38 \%)$ \\
\hline Femenino & $253(54 \%)$ & $1073(57 \%)$ & $2(29 \%)$ & $510(60 \%)$ & $61(55 \%)$ & $441(60 \%)$ & 0 & $538(62 \%)$ \\
\hline Consumo de alimentos en la calle* & $256^{\#}(55 \%)$ & $946(50 \%)$ & $6^{\#}(86 \%)$ & $334(39 \%)$ & $44(60 \%)$ & $328(45 \%)$ & $2(100 \%)$ & $333(38 \%)$ \\
\hline $\begin{array}{l}\text { * Promedio } \\
\neq \text { Frecuencia } \\
\text { \& } p<0.05 \text { entre pacientes con y sin } \\
\# p<0.001 \text { entre pacientes con y sir }\end{array}$ & óstico de & era du & mismo & & & & & \\
\hline
\end{tabular}




\section{Cuadro II}

Características del cuadro clínico en los pacientes con y sin diagnóstico de cólera. Delegación 2 Noreste del Distrito Federal, Instituto Mexicano del Seguro Social, 1995-1998

\begin{tabular}{|c|c|c|c|c|c|c|c|c|}
\hline \multirow[t]{2}{*}{ Características } & \multicolumn{2}{|c|}{1995} & \multicolumn{2}{|c|}{1996} & \multicolumn{2}{|c|}{1997} & \multicolumn{2}{|c|}{1998} \\
\hline & Con cólera & Sin cólera & Con cólera & Sin cólera & Con cólera & Sin cólera & Con cólera & Sin cólera \\
\hline $\mathrm{N}$ úmero & 469 & 1910 & 7 & 850 & 110 & 732 & 2 & 872 \\
\hline \multirow[t]{2}{*}{ Evacua-ciones líquidas* } & $450^{\S}$ & 813 & 7 & 822 & $110^{\S}$ & 703 & 2 & 832 \\
\hline & $(15 \pm 9)^{\#}$ & $(12 \pm 8)$ & $(11 \pm 8)$ & $(12 \pm 8)$ & $(16 \pm 11)^{\#}$ & $(13 \pm 8)$ & $(7 \pm 4)$ & $(12 \pm 8)$ \\
\hline Aspecto de "agua de arroz" & $146^{\#}(31 \%)$ & $260(14 \%)$ & $1(14 \%)$ & $102(12 \%)$ & $41^{\#}(37 \%)$ & $96(13 \%)$ & 0 & $98(11 \%)$ \\
\hline \multirow[t]{2}{*}{ Vómitos* } & $330^{\#}$ & 1044 & 6 & 552 & $89^{\#}$ & 496 & 1 & 632 \\
\hline & $(8 \pm 8)^{\#}$ & $(4 \pm 5)$ & $(4 \pm 4)$ & $(4 \pm 5)$ & $(5 \pm 8)^{\#}$ & $(4 \pm 6)$ & $(25 \pm 2)$ & $(5 \pm 6)$ \\
\hline Espasmos muscula-res ${ }^{\ddagger}$ & $216^{\#}(46 \%)$ & $489(26 \%)$ & $2(29 \%)$ & $193(23 \%)$ & $68 \#(62 \%)$ & $221(30 \%)$ & 0 & $220(24 \%)$ \\
\hline Deshidra-tación en I o II grado & $384^{\#}(82 \%)$ & $1294(68 \%)$ & $5(71 \%)$ & $624(73 \%)$ & $96^{\S}(87 \%)$ & 575 (79\%) & 0 & $578(66 \%)$ \\
\hline Choque $^{\ddagger}$ & $45^{\#}(10 \%)$ & $23(1.2 \%)$ & 0 & $6(0.7 \%)$ & $14^{\#}(1.2 \%)$ & $5(0.7 \%)$ & 0 & $6(0.7 \%)$ \\
\hline Defun-ción ${ }^{\ddagger}$ & $3(0.6 \%)$ & $7(0.4 \%)$ & 0 & $2(0.2 \%)$ & $2(2 \%)$ & $2(0.2 \%)$ & 0 & 0 \\
\hline $\begin{array}{l}* \text { Frecuencia } \\
\text { ₹ Frecuencia, promedio y desviac } \\
\S p<0.05 \text { entre pacientes con y } s \\
\# p<0.001 \text { entre pacientes con y }\end{array}$ & dar en 2 & ras & nismo $=\tilde{n}^{-1}$ & & & & & \\
\hline
\end{tabular}

Rivera Benítez ${ }^{14}$ en el Hospital General de México, señalan un mayor pocentaje del sexo masculino; sin embargo, en ningún caso se puede considerar el sexo como factor asociado con la enfermedad.

Como agente causal destacó la presencia de $V$. cholerae 01 Ogawa, mientras que en los estudios del Hospital Infantil de México Federico Gómez y del Hospital General de México ${ }^{14}$ predominó $V$. cholerae 01 Inaba, cambio epidemiológico ya señalado por Giono ${ }^{9}$ y Sánchez ${ }^{8}$ tanto en México como en América Latina.

Respecto al cuadro clínico se observa que, aun cuando presentaron variación en su gravedad, prácticamente todos los casos de cólera fueron sintomáticos, lo que contrasta con otras fuentes que mencionan hasta $75 \%$ de infecciones asintomáticas. ${ }^{3}$ Esta diferencia quizá podría explicarse a partir de un estudio realizado por Glass, ${ }^{15}$ quien afirma que la proporción de la población que presenta diarrea grave secundaria a cólera es mucho mayor en América que en Asia, debido a que en el primer caso la población no fue previamente inmunizada por la infección y porque, además, presenta una mayor prevalencia del grupo sanguíneo O positivo, el cual es un factor predisponente a la enfermedad severa; cabe aclarar que en este estudio el grupo sanguíneo no fue una variable investigada.

Al comparar las características de los casos y los no casos, es notable que en los años de mayor inciden- cia, tanto la frecuencia como la gravedad de los signos y síntomas hayan sido mayores entre los pacientes con cólera que entre aquellos con diarrea de otro origen. Así, las heces con aspecto de "agua de arroz" se presentaron en 30-40\% de los pacientes, porcentaje mayor al referido por la Secretaría de Salud, ${ }^{3}$ pero menor al mencionado en los estudios de Lázaro Cárdenas, en Michoacán ${ }^{11}$ y de Huautla de Jiménez, en Oaxaca, ${ }^{13}$ quizá porque en ambos se hace referencia a una epidemia explosiva, mientras que en este estudio predominaron los casos aislados. Llama la atención que $14 \%$ de los pacientes con diarrea de otro origen hayan referido estas evacuaciones, y no hay una explicación clara al respecto; quizá se trataba de portadores de cólera no detectados por errores en la toma de la muestra, o puede ser que los cultivos hayan resultado negativos debido a la ingesta previa de antibióticos, o bien, que las heces no hayan sido realmente como "agua de arroz", pero que se describieron así a causa de un interrogatorio inducido.

Similar a lo encontrado en otros estudios,,$^{2,11-14}$ en esta investigación la deshidratación fue la complicación más frecuente y afectó más a los pacientes con cólera que a aquellos con diarrea de otro origen, situación todavía más evidente en el caso del choque hipovolémico; este último se presentó, sin embargo, en una menor proporción respecto a los otros estu- 
dios, quizá porque el mayor conocimiento de la enfermedad ha permitido a los servicios de salud actuar en forma más adecuada y oportuna.

Con base en todo lo anterior, podemos considerar que la presencia y la gravedad de ciertos datos clínicos facilita al médico establecer el diagnóstico de cólera en las épocas en que la enfermedad es frecuente, no así cuando es poco común, ya que entonces los datos clínicos no son característicos.

En relación con la mortalidad, las cifras en los pacientes con cólera de este estudio oscilaron entre $0 \mathrm{y}$ $2 \%$, con un promedio de $0.87 \%$; aunque menores a otras, ${ }^{2,11-14}$ durante 1997 estas proporciones fueron superiores al $1 \%$ señalado por la Secretaría de Salud como parámetro aceptable. Aunque no se especifica en los resultados, cabe mencionar que los pacientes fallecidos fueron en general aquellos que tenían características especiales (edad avanzada y/o enfermedades crónico-degenerativas), de tal forma que la deshidratación facilitó la presencia de complicaciones más severas, como lo describen Rivera Benítez ${ }^{14}$ y Rodríguez López ${ }^{12}$ en otros trabajos.

Finalmente, podemos concluir que el cólera muestra un comportamiento cíclico bianual, tanto en la frecuencia como en la gravedad de los casos; y aunque la presencia del padecimiento tiende a disminuir, se debe continuar e incluso incrementar la vigilancia sanitaria y epidemiológica, así como el esfuerzo del médico para establecer de manera oportuna un diagnóstico y un tratamiento adecuado.

\section{Referencias}

1. C ampollo-Rivas 0. Cólera: historia, epidemiología, diagnóstico y manejo clínico. Rev Gastroenterol Mex 1992;57(1):45-51.

2. Lezama-Basulto LA, Mota-Hernández F,Bravo-Barrios E. Cólera en niños. Informe de 8 casos. Bol Med Hosp Infant Mex 1993;50:789-796.

3. Manual para la vigilancia epidemiológica del cólera en México. México, D.F: Dirección General de Epidemiología-Subsecretaría de Coordinación y D esarrollo-Secretaría de Salud, 1992:13-16.

4. Lezama-Basulto LA, Mota-Hernández F. Cólera en pediatría. Bol Med Hosp Infant Mex 1993;50:671-677.

5. Mota-Hernández F. Las enfermedades diarreicas y el cólera. Bol Med Hosp Infant Mex 1992;49:809-812.

6. Boletín Epidemiológico del Cólera 1991-1998. Dirección General de Epidemiología-Secretaría de Salud.

7. Boletín Epidemio lógico A nual 1991-1998. Jefatura de Servicios de SaludSubdirección General Médica-Instituto Mexicano del Seguro Social.

8. Sánchez JL, Taylor D N . C holera. Lancet 1997;349:1825-1829.

9. Giono S, Gutiérrez-Cogno L, Rodríguez-Angeles G, Del Río-Zolezzi A, Valdespino-G onzález JL, Sepúlveda-Amor J. Vibro cholerae 01 fenotipos y genotipos México. Gac Med Mex 1995;131:28-37.

10. Jiménez-C orona A, G utiérrez-C ogio L, López-Moreno S,Tapia-C onyer R. El cólera en México. Situación epidemiológica actual. Gac Med Mex 1995;131:363-366.

11. Pastrana-H umnaco E,A barca-Sánchez V, Herrera-Rivas G, RodríguezA révalo J. Epidemia de cólera en Lázaro Cárdenas, Michoacán. Rev Med IMSS 1994:32:259-261.

12. Rodríguez-López L, Rubio-G uerraA, Lozano-N uevo JJ,Vargas-Ayala G . Cólera en el anciano. Med Int Mex 1995;11:56-58.

13. Sánchez-Barajas P,A riza-Andraca R, Frati-Munari A.Cólera en la Sierra Mazateca. A nálisis de 80 casos. Rev Med IMSS 1992;30:369-371.

14. Rivera-Benítez C, Solache-Alcaraz G, C uauthli-Espinosa de los Monteros M, Higuera Ramírez F. Experiencia clínica del cólera en el Hospital General de México. Med Int Mex 1993;9:93-96.

15. G lass RI, Libel M, Brandling-Bennet AD. Epidemic cholera in the Americas. Science 1992;256:1524-1525. 